\title{
Elaboração de material disseminável acerca da conduta de enfermeiros frente aos eventos adversos pós-vacinação para apoio aos docentes em saúde
}

\section{Development of material to disseminate about the conduct of nurses to the adverse events post-vaccination for support for teachers in health}

\author{
${ }^{1}$ Mariane de Paula Gomes \\ ${ }^{2}$ Maria de Fátima Alves de Oliveira \\ ${ }^{3}$ Fábio Aguiar Alves
}

\begin{abstract}
RESUMO
No presente trabalho propõe-se a elaboração de material disseminável acerca da conduta de enfermeiros frente aos eventos adversos pós-vacinação, na forma de recurso tecnológico, para orientação/complementação/apoio aos docentes nas disciplinas de imunologia, microbiologia, saúde coletiva e áreas afins, visando à orientação e obtenção de respostas rápidas às dúvidas que possam acontecer quando da execução do trabalho de imunização, principalmente no que concerne aos Eventos Adversos Pós-Vacinação. O interesse pela temática surgiu da necessidade de criar subsídios que auxiliem os docentes no processo de ensino-aprendizagem de acadêmicos de enfermagem acerca dos imunobiológicos ofertados no Programa Nacional de Imunização. Pesquisa descritiva, de abordagem quanti-qualitativa e caráter exploratório, os dados foram coletados em um curso de graduação em Enfermagem no município de Volta Redonda/RJ, a partir de dois questionários aplicados aos docentes. Os resultados apontaram que os docentes demonstraram-se motivados com a possibilidade de ter acesso ao recurso tecnológico de apoio, já que poderá oferecer suporte às atividades pedagógicas e de aprendizado. Outro aspecto importante foi o reconhecimento por parte dos docentes quanto à utilização do site elaborado, evidenciado a partir do processo de avaliação e validação do produto. Nesse sentido, acredita-se que a utilização do site como ferramenta, possibilitará mudanças de comportamento na relação docente/discente, além de melhorias na prática profissional tanto dos docentes, a partir da diversificação dos métodos de ensino, quanto dos discentes a partir da adoção de condutas mais eficientes e condizentes quanto ao do processo de imunização e dos possíveis eventos que por ventura possam surgir.
\end{abstract}

Palavras-chave: ensino de enfermagem; imunização; eventos adversos pós-vacinação; recurso tecnológico.

\section{ABSTRACT}

In this paper we propose the development of materials to disseminate about the conduct of nurses to the adverse events post-vaccination in the form of technological resource for orientation/complementation/ support for teachers in the discipline of immunology, microbiology, collective health and related areas,

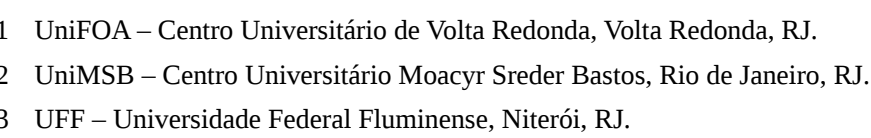


targeting the orientation and obtaining quick answers to any questions that may occur when executing the immunization work, especially with regard to the Adverse Events post vaccination. The interest in the area arose from the need for create subsidies to assist teachers in the teaching-learning of nursing students about immunobiological offered in the National Immunization Program. Descriptive search, quantitativequalitative approach and exploratory, the data were collected on graduate course in nursing in the city of Volta Redonda/RJ, from two questionnaires given to teachers. The results showed that teachers proved to be motivated with the possibility of having access to technological support resource as it may support the teaching and learning activities. Another important aspect was the recognition by the teachers on the use of elaborate site, evidenced from the process of evaluation and validation of the product. In this sense, it is believed that the use of the site as a tool for guidance/complementation/support about adverse events post-vaccination, presented here, enable, above all, behavioral changes in a teacher/student ratio, and improvements in both professional practice of teachers, the from the diversification of teaching methods, how students, in adoption of more efficient and consistent behaviors regarding the immunization process and possible events that may eventually arise.

Keywords: Teaching nursing; Immunization; Adverse events post vaccination; Technological resource.

\section{INTRODUÇÃO}

As especificidades e o aparato envolvido na administração de cada vacina fazem do Programa Nacional de Imunização, um programa complexo e por isso exige tanta responsabilidade. Erros na aplicação da técnica podem causar sérios eventos adversos. Os eventos adversos pós-vacinação são definidos pelo Manual de vigilância epidemiológica de eventos adversos pós-vacinação (2008, p. 24), como: "Qualquer ocorrência clínica indesejável em indivíduo que tenha recebido algum imunobiológico (...)”.

O desenvolvimento de um recurso tecnológico como instrumento didático de ensino sobre eventos adversos pós-vacinação, favorece aos docentes, não só os de enfermagem, como também os das demais áreas da saúde, permitindo maior possibilidade de orientação em relação aos conteúdos, além de otimização do processo. Por mais explicativos que a bibliografia e/ou manuais sejam, ainda assim não o fazem como instrumento único e facilitador do aprendizado. Desta forma, além de contribuir para minimizar as dúvidas e facilitar a assimilação dos conteúdos pelos acadêmicos de enfermagem, contribui para o desenvolvimento de uma assistência eficiente e de qualidade.

\section{OBJETIVOS}

A partir do desenvolvimento do recurso tecnológico o presente estudo objetiva: apresentar os Eventos Adversos Pós-vacinação, a partir da utilização de um recurso tecnológico, no ensino de graduação em enfermagem e; estimular a utilização da ferramenta para orientação acerca dos Eventos Adversos Pós-Vacinação associados ao uso de vacina, bem como a conduta para os eventos que por ventura possam surgir.

\section{METODOLOGIA}

Trata-se de uma pesquisa descritiva, de abordagem quanti-qualitativa e caráter exploratório, onde os dados coletados foram obtidos a partir de dois questionários.

O cenário escolhido foi um curso de graduação em Enfermagem de uma instituição de ensino superior privada do município de Volta Redonda, RJ. O estudo de campo iniciou após aprovação do Comitê de Ética e 
Pesquisa em Seres Humanos - CoEPS, do Centro Universitário de Volta Redonda - UniFOA, sob parecer n ${ }^{\circ}$ 139.762 em 06 de novembro de 2012. Foram aplicados dois questionários aos docentes do curso de enfermagem - sujeitos da pesquisa - que assinaram previamente o Termo de Consentimento Livre e Esclarecido (TCLE).

Na coleta de dados foi aplicado, num primeiro momento, um questionário com perguntas abertas e fechadas, antes da apresentação do recurso tecnológico sobre eventos adversos pós-vacinação com o objetivo de investigar o perfil/concepção dos docentes acerca da utilização do produto.

Posteriormente a apresentação do produto, o segundo questionário foi aplicado, objetivando avaliar a qualidade do material produzido, quanto à interface (layout) do produto e; quanto ao conteúdo apresentado.

\section{ANÁLISE E DISCUSSÃO DOS DADOS}

\section{1 Análise do Primeiro Questionário - Perfil/concepções dos docentes acerca da utilização do produto}

A primeira coleta de dados ocorreu no mês de novembro de 2012, sendo a amostra composta por vinte e um dos trinta e nove que integravam o corpo docente neste período. Dos que responderam ao questionário, dezesseis eram do gênero feminino e cinco do masculino. Confirmando o contexto histórico da Enfermagem marcado pela supremacia da força de trabalho feminina no desenvolvimento de tarefas que envolvem o cuidado no Brasil e no mundo.

De acordo com a graduação/formação dos docentes envolvidos na pesquisa, observou-se que há uma diversificação de profissionais atuando no curso de enfermagem, o que pode ser justificado a partir das disciplinas generalistas exigidas na grade curricular. Destacou-se também o fato de apenas um profissional ter menos de cinco anos de formação, o que manifesta por parte do Curso de Enfermagem certa diligência quanto à valorização dos profissionais com experiência para atuarem no processo de ensino-aprendizagem.

Thiesen em seus estudos (2008, p. 546) destaca a interdisciplinaridade, "como um movimento contemporâneo que emerge na perspectiva da dialogicidade e da integração das ciências e do conhecimento, vem buscando romper com o caráter de hiperespecialização e com a fragmentação dos saberes”.

De acordo com os itens: formação pós-graduação e atuação profissional respectivamente nove são especialistas, dez são mestres e dois são doutores. No momento da coleta de dados a grande maioria dos docentes buscava atualização, capacitação e aprimoramento da formação acadêmica.

Com o intuito de colocar no mercado profissionais autônomos e com capacidade crítica, o curso investe na formação generalista. E vários docentes lecionam durante o semestre mais de uma disciplina dentre básicas e específicas reforçando ainda mais a interdisciplinaridade. Para Rocha e Rocha (2013, p. 6) "a proposta interdisciplinar propõe uma profunda revisão de pensamento, no sentido da intensificação do diálogo, da integração conceitual e metodológica nos diferentes campos do saber”.

Ao serem questionados quanto aos recursos didáticos utilizados em suas disciplinas, com exceção de um professor, que relatou não utilizar por atuar em campo de estágio, de uma forma geral, todos os docentes fazem uso de mais de tipo de recurso didático e, de acordo com alguns relatos, quando utilizados, servem como apoio para nortear e favorecer a assimilação de conteúdos.

Os recursos ou tecnologias educacionais, também conhecidos como materiais e equipamentos didáticos são entendidos e definidos por Freitas (2007, p. 21), como "todo e qualquer recurso utilizado em um procedimento de ensino, visando à estimulação do aluno e à sua aproximação do conteúdo”. 
A inserção de novos meios como auxiliares na educação é um dos vários motivos que favorece estas mudanças nas atividades práticas. Para Bernini, et. al. (2009, p.3): “A expressão ensino-aprendizagem passa a ter nova conotação, antes se entendia: alguém ensina para alguém aprender”, hoje se entende: "alguém organiza atividades e orienta para que alguém interaja com as informações”.

Ao analisar o discurso dos docentes de enfermagem quanto à utilização de um recurso tecnológico voltado para conteúdos específicos, buscou-se identificar a partir dos elementos apresentados, aqueles com maior aproximação/semelhança nas respostas, ou seja, quanto à mesma natureza, mesma função, ou mesmo efeito e, assim elaborar os resultados.

De acordo com Bardin (2011, p. 135): “Fazer uma análise temática consiste em descobrir os “núcleos de sentido" que compõem a comunicação e cuja presença, ou frequência de aparição, podem significar alguma coisa para o objetivo analítico escolhido”.

Os agrupamentos formados pela similaridade das respostas resultaram nas seguintes categorias: $\mathrm{O}$ recurso tecnológico como ferramenta de apoio para o aprimoramento do ensino e; O recurso tecnológico como diversificação de recurso didático.

\section{20 recurso tecnológico como ferramenta de apoio para o aprimoramento do ensino}

Os resultados apontaram que dos vinte e um docentes, seis afirmaram que o recurso tecnológico pode funcionar como ferramenta auxiliar no aprimoramento do ensino. A utilização da tecnologia como instrumento/ ferramenta, talvez seja a maneira mais importante considerando os aspectos de ensino e aprendizagem. Uma vez empregados e disponibilizados no ensino servem como complementos da metodologia, sendo os recursos utilizados pelos alunos e pelo professor para organizar de forma metódica o processo ensino-aprendizagem (ÁVILA, 2009; PRAMIU ET. AL., 2011).

Alencar e Fernandes (2010) apontam em seus estudos acerca do emprego de ferramentas computacionais como recurso de apoio didático-pedagógico, que repensar o uso de tecnologia nos ambientes educacionais torna-se obrigatório a todos os professores do século XXI, bem como para a sociedade, já que o foco do processo educativo é alterado passando do professor para o aluno. A utilização do recurso tecnológico/computador como ferramenta complementar e de apoio ao ensino, acaba por estimular o aluno à participação ativa, à experimentação, à colaboração e a autoconstrução de seu conhecimento.

Não se pode negar a importância do uso de novas tecnologias, principalmente na área da informática na atual conjuntura da sociedade. Desta forma entende-se que o recurso tecnológico como recurso didático, pode subsidiar e auxiliar o processo de ensino-aprendizagem podendo sim, ser uma ferramenta de apoio às aulas, favorecendo nesse sentido, ao aprimoramento do processo de ensino-aprendizagem.

\section{3 O recurso tecnológico como diversificação de recurso didático}

Assim como Falkembach (2005) sinaliza que o uso da informática possibilitou a utilização do computador e outros recursos multimídias como auxiliares no processo de ensino-aprendizagem, destacando a necessidade de adaptação de novos recursos tecnológicos na educação, Neves e outros autores, (2010), também apontam que o uso integrado de tecnologias de informação e multimídia são capazes de proporcionar melhorias significativas no processo ensino e aprendizagem, uma vez que permite ao professor possibilidades de expor e construir conteúdos, num modelo de ensino centrado principalmente no aluno.

De acordo com o relato de cinco dos vinte e um professores, o recurso tecnológico quando utilizado como recurso didático, além de tornar o processo de ensino dinâmico, favorece nas formas de comunicação do conhecimento. 
Para Silva e Pessoa (2012, p.2):

A utilização de recursos variados implica ensino com pesquisa, atitude mediadora de educadores, que pautados na ética levam seus educandos a serem agentes da educação e apreenderem atitudes necessárias para o desenvolvimento e autonomia na aprendizagem, ensinando-os atuarem de modo crítico e transformador na sociedade em que estão inseridos.

Segundo Moraes (2006, p.04) “a postura do professor crítico é conhecer a nova realidade formatada pelas tecnologias de informação e comunicação na sociedade e aceitar as mudanças”. Paulo Freire (2010) já alertava quanto à aceitação do diferente e, como professor se considerava um “aventureiro” responsável predisposto à mudança.

Assim, pode-se considerar que o processo de ensino-aprendizagem foi alterado a partir do advento e principalmente do avanço tecnológico ocorridos nos últimos anos. O fácil acesso aos computadores e a internet, permitiu tanto aos estudantes quanto aos professores, novos recursos tecnológicos que quando inseridos no ambiente de ensino-aprendizagem favorecem na construção do conhecimento pelo alunado e a novas técnicas didático-pedagógicas aos professores durante as aulas.

A partir da análise das falas ficaram ainda evidenciados outros benefícios da utilização do recurso tecnológico na prática dos docentes, como a assimilação/fixação de conteúdos, a atualização do docente e, a otimização do serviço.

Evidencia-se que a utilização de novas tecnologias é praticamente ilimitada e têm papel fundamental na educação e também no desenvolvimento social e cultural, já que influenciam diretamente toda a sociedade. Quando inseridos nos espaços e sistemas educativos como ferramentas pedagógicas de apoio, tornam-se essenciais uma vez eu assumem um novo método de transmissão de conhecimentos.

Diante desse contexto, pode-se afirmar que o professor tem por incumbência gerenciar todas as novas ferramentas que tem ao seu dispor de forma a facilitar a sua vida e principalmente a vida dos educandos, favorecendo assim uma mudança de paradigma quanto à informação e formação dos mesmos.

\section{4 Análise do Segundo Questionário - Instrumento de Validação do Produto}

O segundo questionário foi respondido por doze dos vinte docentes do curso de enfermagem que estiveram presentes na apresentação do produto dia 19 de novembro de 2013. Após a apresentação todos receberam o questionário e tiveram o prazo de uma semana para acessarem o site e avaliar a qualidade do material produzido quanto à interface (layout) do produto (Organização, disponibilidade e legibilidade das informações) e; quanto ao conteúdo apresentado (Suficiência, pertinência, objetividade, eficiência).

A interface é o meio que torna possível o caminho de acesso ou de ligação entre o usuário e a aplicação. No âmbito deste trabalho, a aplicação baseia-se em uma página virtual destinada à educação. Trata-se de um programa/site para orientação/complementação/apoio aos docentes nas disciplinas de imunologia, microbiologia, saúde coletiva e áreas afins, acerca dos Eventos Adversos Pós-Vacinação.

Quanto à avaliação geral do site, nove (75\%) avaliadores emitiram a opinião excelente ao passo que apenas três (25\%) docentes avaliaram o site como muito bom. Os itens de avaliação regular e ruim não foram assinalados em nenhum quesito do questionário. Enquanto o item bom apareceu por duas vezes, nos itens aparência estética e navegabilidade do site.

Para os avaliadores, de modo geral, o site apresenta excelente aparência, excelente estrutura de navegação e usabilidade, tem aplicabilidade no processo de ensino, além de servir também como material de apoio e atuali- 
zação aos egressos. Apresenta imagens e links interligando textos, recursos gráficos e textuais e torna possível aos usuários realizar buscas acerca de diferentes conteúdos educacionais, visto que contempla diferentes disciplinas.

Os conteúdos atendem aos requesitos de suficiência, pertinência, objetividade e eficiência, podendo trabalhar com conteúdos restritos, delimitado a uma disciplina especificamente ou mesmo trabalhar concorrentemente as mais diversas áreas do conhecimento. Por essa razão, os docentes afirmaram utilizar e indicar aos discentes como material complementar ao processo de ensino-aprendizagem.

Como a aceitação de um sistema interativo é determinante do sucesso do sistema, o fator satisfação do usuário enfatiza a avaliação subjetiva do sistema feita por seus usuários, incluindo emoções que possam surgir durante a interação, sejam elas positivas, como prazer e diversão, ou negativas, como tédio ou frustração (PRATES E BARBOSA, 2003, p.248).

O produto foi validado com excelência e sua real intenção educacional foi evidenciada. Uma vez que integra particularidades do conteúdo com variáveis, como a interatividade e a comunicação através da internet e o público-alvo, sendo pertinentes aos docentes como material de apoio as disciplinas, aos discentes no auxílio às pesquisas e aprofundamento do conhecimento e até mesmo, aos demais usuários, com respostas a diversos questionamentos.

\section{CONCLUSÃO}

É notável que as práticas e os cuidados em saúde nas últimas décadas passaram por profundas mudanças e alterações num contexto de diversidades e complexidade. No ensino não é diferente, o uso da tecnologia como recurso didático, têm sinalizado e apontado melhorias no processo de ensino-aprendizagem. Uma maneira de tornar moderno e ampliar o vínculo entre a sociedade e a escola, atendendo as atuais demandas educacionais, é dispor a utilização das tecnologias que se tem acesso à informação aos professores e alunos.

Tendo como base a proposta de desenvolver um programa abrangente dos Eventos Adversos Pós-Vacinação como instrumento de ensino nas condutas do cuidar para acadêmicos de enfermagem e, considerando o perfil/concepções dos docentes acerca da utilização do produto, observa-se que se torna imprescindível ao acadêmico conhecer e aprender sobre os possíveis eventos adversos pós-vacinação e como conduzi-los, uma vez que em breve durante sua assistência, poderá vivenciar as dificuldades em seu dia a dia relacionado a esta atividade.

Informações acerca dos eventos adversos e tudo o que se refere à imunização dos seres humanos, são oportunas e bem-vindas para os acadêmicos, profissionais e principalmente para os usuários do sistema de saúde brasileiro.

Os resultados apontaram que os docentes que participaram do estudo, demonstraram-se motivados e colaborativos com a possibilidade de ter acesso ao recurso tecnológico de apoio, já que poderá oferecer suporte às atividades pedagógicas e de aprendizado, objetivando qualidade, flexibilidade e individualização no processo de aprendizagem.

Outro aspecto importante do estudo foi o reconhecimento por parte dos docentes quanto à utilização do site elaborado, onde sua aceitação foi evidenciada a partir do processo de avaliação e validação do produto. Por se tratar de um material voltado para fins educacionais, todos os recursos nele disponibilizado possibilitam aos usuários buscar variados conteúdos, visto que contempla diferentes disciplinas. Portanto, o mesmo pode ser utilizado como recurso didático relevante à aprendizagem, uma vez que favorece aos alunos maior entendimento e consequentemente a construção do conhecimento, além de viabilizar também aos docentes e profissionais da área da saúde, principalmente aos enfermeiros, suporte informativo e atualização constante no que concerne à temática. 
Cabe ressaltar, que o site tem por objetivo auxiliar o trabalho do docente não interferindo na autonomia do educando já que não limita a totalidade do processo de aprendizagem servindo como um instrumento importante para o discente no aprofundamento e assimilação do conhecimento.

Nesse sentido, acredita-se que a utilização do site como ferramenta para orientação/complementação/ apoio acerca dos eventos adversos pós-vacinação, aqui apresentado, possibilitará acima de tudo, mudanças de comportamento na relação docente/discente, além de melhorias na prática profissional tanto dos docentes, a partir da diversificação dos métodos de ensino, quanto dos discentes a partir da adoção de condutas mais eficientes e condizentes quanto ao do processo de imunização e dos possíveis eventos que por ventura possam surgir.

\section{REFERÊNCIAS}

ALENCAR, W. S. \& FERNANDES, R. F. Emprego de ferramentas computacionais como recurso de apoio didático-pedagógico para o magistério de matemática e Ciências no Ensino Fundamental. In: Anais do XXI Simpósio Brasileiro de Informática na Educação - SBIE. João Pessoa - PB, 2010.

ÁVILA, V. A. Desenvolvimento de uma ferramenta audiovisual aplicada ao ensino da farmacologia. Volta Redonda: UniFOA, 2009. Dissertação (Mestrado Profissional) - Centro Universitário de Volta Redonda UniFOA. Pós-graduação em Ensino em Ciências da Saúde e do Meio Ambiente, 2009.

BARDIN, L. Análise de conteúdo. São Paulo: Edições 70, 2011.

BRASIL. Ministério da Saúde. Secretaria de Vigilância em Saúde. Departamento de Vigilância Epidemiológica. Manual de vigilância epidemiológica de eventos adversos pós-vacinação. Brasília: Ministério da Saúde, 2008.

FALKEMBACH, G. A. M. Concepção e desenvolvimento de material educativo digital. Novas Tecnologias na Educação - CINTED - UFRGS V.3 Nº 1, Maio, 2005.

FREIRE, P. Pedagogia da Autonomia: saberes necessários à prática educativa. $41^{\mathrm{a}}$ ed. São Paulo: Paz e Terra, 2010.

FREITAS, O. Equipamentos e materiais didáticos. - Brasília: Universidade de Brasília, 2007.

MORAES, S. A de. $\mathbf{O}$ uso da internet na prática docente: reflexões de uma pesquisadora em ação. Universidade Estadual de Maringá. Programa de pós-graduação em educação: Mestrado. Área de concentração: aprendizagem e ação docente. Maringá, 2006.

NEVES, A. dos S. Desenvolvimento de software: sistema informatizado de educação nutricional via web. Volta Redonda: UniFOA, 2010. Dissertação (Mestrado Profissional) - Centro Universitário de Volta Redonda UniFOA. Pós-graduação em Ensino em Ciências da Saúde e do Meio Ambiente, 2010.

PRAMIU, P. V.; PRADO, N. V.; RIZZI, R. L. \& RIZZI, C. B. O emprego do software Maxima como ferramenta de apoio na formação continuada de professores de matemática: do relato do curso a considerações sobre seus desdobramentos. In: Encontro Nacional de Informática e Educação. UNIOESTE, Campus Cascavel - PR, 2011.

PRATES, R. O. ; BARBOSA, S.D.J. Avaliação de Interfaces de Usuário - Conceitos e Métodos. In: Juan Manuel Adán Coello; Sandra C. P. Ferraz Fabbri. (Org.). Jornada de Atualização em Informática do Congresso da Sociedade Brasileira de Computação. Campinas: SBC, 2003, v. 2, p. 245-293. 
ROCHA, S. de F. M.; ROCHA, J. H. de M. V. A interdisciplinaridade em Paulo Freire: Reflexões em defesa do diálogo disciplinar na educação. Resumos do VIII Colóquio Internacional Paulo Freire. Centro Paulo Freire - Estudos e Pesquisas: Recife, 2013.

SILVA, A. M.; PESSOA, M. P. Recursos Didáticos e Inovações Tecnológicas no Ensino de Língua Estrangeira Moderna, 2012. Disponível em: http://www.diaadiaeducacao.pr.gov.br/portals/pde/arquivos/1452-8.pdf Acesso em: 10/09/2013.

THIESEN, J. S. A interdisciplinaridade como um movimento articulador no processo ensino-aprendizagem. Revista Brasileira de Educação v. 13 n. 39 set./dez. 2008. 